\title{
Tipos de Fibras no Músculo Flexor Longo do Hálux de Frangos de Corte Submetidos ao Estresse pelo Calor e Frio e Alimentados em "Pair-Feeding"1
}

\author{
José Roberto Sartori ${ }^{2}$, Elisabeth Gonzales ${ }^{3}$, Marcos Macari ${ }^{4}$, Vitalino Dal Pai ${ }^{5}$, \\ Henrique Nunes de Oliveira 6
}

\begin{abstract}
RESUMO - O objetivo deste trabalho foi avaliar o efeito da temperatura ambiente sobre as características morfométricas das fibras musculares do músculo flexor longo do hálux de frangos de corte alimentados com a mesma quantidade de ração ("pair-feeding"), isolando-se o efeito do consumo voluntário de alimento. Foram utilizados 16 frangos de corte machos, Ross, distribuídos em um delineamento inteiramente casualizado com dois tratamentos (estresse pelo calor e frio), com sete e nove repetições cada, respectivamente. Aos 43 dias de idade, o peso médio das aves estressadas pelo calor (1255 g) foi maior que o das aves estressadas pelo frio em "pair-feeding" (1086 g). O músculo das aves submetidas ao estresse pelo calor e pelo frio apresentou 22,82 e $27,93 \%$ de fibras brancas (FG - Fast Glycolytic), 52,76 e 47,67\% de intermediárias (FOG - Fast Oxidative Glycolytic) e 24,42 e 24,40\% de vermelhas (SO - Slow Oxidative), respectivamente. O diâmetro das fibras FG foi maior no músculo das aves submetidas ao calor (48,69 $\mathrm{mm})$, quando comparado ao das aves submetidas ao frio $(37,74 \mathrm{~mm})$. A freqüência e o número dos tipos de fibras no músculo não diferiram entre as aves estressadas pelo calor e pelo frio. O estresse pelo frio associado à limitação no consumo de alimento determinou redução no crescimento e alterou a composição dos tipos de fibras no músculo flexor longo do hálux de frangos de corte, reduzindo o tamanho das fibras do tipo FG e mantendo o tamanho das fibras SO e FOG, as quais apresentam metabolismo oxidativo, associado à maior produção de calor.
\end{abstract}

Palavras-chave: estresse, fibra, frangos de corte, músculo esquelético, temperatura

\section{Fiber Types of Flexor Hallucis Longus Muscle in Pair-Fed Broilers under Heat and Cold Stress}

\begin{abstract}
This experiment aimed to evaluate the effect of environmental temperature upon the morphometric characteristics of the fiber types in the flexor hallucis longus muscle of broilers in pair-feeding, thus isolating the effect of voluntary feed intake. Sixteen male broiler, Ross, were allotted to a completely randomized design with two treatments (heat stress and cold stress), and seven and nine replications, respectively. Forty-three-day-old heat stressed broilers weighed $1.255 \mathrm{~g}$ and those in cold stress, pair-fed, weighed $1.086 \mathrm{~g}$. The birds subjected to heat and cold environmental temperatures showed 22.82 and 27.93\% white (FG - Fast Glycolytic), 52.76 and $47.67 \%$ intermediate (FOG - Fast Oxidative Glycolytic) and 24.42 and $24.40 \%$ red (SO - Slow Oxidative) fibers in the flexor hallucis longus muscle, respectively. The diameter of FG fiber was higher in the muscle of heat stressed birds (48.69 mm), comparing to cold stressed pair-fed ones $(37.74 \mathrm{~mm})$. Temperature had no effect on the number and frequency of fibers. The cold stress associated with restricted feed intake depressed growth and reduced the size of FG fibers in the flexor hallucis longus muscle of broilers, but did not affect the size of FOG and SO type fibers, which have higher oxidative metabolism which is associated with heat production.
\end{abstract}

Key Words: broilers, pair-feeding, skeletal muscle, stress, temperature

\section{Introdução}

A maioria dos países do mundo tem apresentado expressivo aumento no consumo de carne de aves na última década, com os frangos de corte contribuindo com $70 \%$ do total da carne de aves consumida. Nas próximas décadas, a avicultura continuará sendo uma das maiores opções de fornecimento de carne no mundo (Roenigk, 1999). O uso da carne de peito e pernas das aves, como matéria-prima para elaboração de produtos que atendam a necessidade dos consumidores por carne desossada e produtos elaborados e prontos para o consumo, é cada vez maior (Newcombe, 1994). Considerando que "carne" corresponde à musculatura esquelética das aves, os estudos do crescimento das aves devem avaliar as

\footnotetext{
1 Parte da Tese de Doutorado apresentada à FCAV, UNESP, Jaboticabal, SP, pelo primeiro autor. Apoio: FAPESP

2 Prof. Assistente Dr., Depto. Melhoramento e Nutrição, FMVZ, UNESP, Botucatu, SP. E.mail: jrsartori@fca.unesp.br

3 Prof. Dr. Voluntário, Depto. Clínica Veterinária, FMVZ, UNESP, Botucatu, SP. E.mail: elisa.gonzales@uol.com.br

6 prof. Titular do Depto. Morfologia e Fisiologia, FCAV, UNESP, Jaboticabal, SP. E.mail: macari@fcav.unesp.br

4 Prof. Titular do Depto. Morfologia e Embriologia, IB, UNESP, Botucatu, SP.E.mail: btdalpai@zaz.com.br

5 Prof. Dr. do Depto. Melhoramento e Nutrição, FMVZ, UNESP, Botucatu, SP. E.mail: hnunes@fca.unesp.br
} 
mudanças nos músculos e as influências no seu uso como carne, de modo que técnicas que aumentam o crescimento muscular e alteram de maneira indesejável a qualidade da carne possam ser identificadas e evitadas (Sams, 1999).

Segundo Dubowitz \& Brooke (1984), três tipos de fibras podem ser identificadas nas musculaturas das aves, demonstradas por técnicas histoquímicas, tipo I - contração lenta e oxidativa; tipo IIA - contração rápida e oxidativa e glicolítica; e tipo IIB - contração rápida e glicolítica, as quais correspondem às fibras SO (Slow Oxidative), FOG (Fast Oxidative Glycolytic) e FG (Fast Glycolytic), respectivamente, pela nomenclatura de Peter et al. (1972). As fibras do tipo I são pequenas e têm numerosas mitocôndrias e o pigmento mioglobina em abundância, o que lhes confere a cor vermelha. As fibras do tipo II são células musculares grandes que possuem pequena quantidade de mioglobina e de mitocôndrias. As fibras do tipo IIA são resistentes à fadiga, enquanto as fibras IIB são facilmente fatigáveis, com grande acúmulo de ácido lático. As fibras aeróbicas, por estarem associadas a processo contínuo de produção e consumo de energia, têm elevada troca de metabólitos e oxigênio, tendo pequena área. Já as fibras anaeróbicas, com reduzida taxa de trocas metabólicas e de oxigênio, apresentam maior área e, portanto, maior processo de hipertrofia (Banks, 1992).

Alterações na composição da musculatura esquelética das aves, relacionadas com número, tamanho e freqüência dos diferentes tipos de fibras musculares, podem influenciar a conservação e as características organolépticas da carne, por interferir nos eventos pós-morte durante a conversão do músculo em carne (Chambers et al., 1989; Sams, 1999).

Entre os vários fatores que podem afetar a composição dos tipos de fibras na musculatura esquelética das aves, como o tamanho e a freqüência, estão a nutrição e a temperatura ambiente (Dauncey \& Gilmour, 1996). Vários autores têm relatado aumento na atividade oxidativa e na proporção de fibras oxidativas na musculatura esquelética das aves submetidas ao frio (Duchamp et al., 1992) e de suínos submetidos ao frio e com baixo nível de ingestão de energia (Ingram \& Dauncey, 1986; Dauncey \& Ingram, 1988; Harrison et al., 1996). O oposto foi relatado por Ballantyne \& George (1978), os quais verificaram que a aclimatação ao frio resultou em diminuição no tamanho das fibras musculares vermelhas do músculo peitoral de pombos aclimatados ao frio, sem que se observem alterações nas fibras brancas. Outros músculos esqueléticos analisados em pombos aclimatados ao frio tiveram decréscimo menor e não-significativo no diâmetro das fibras. Todavia, os músculos dos pombos aclimatados ao calor não apresentaram alterações no diâmetro das fibras.

Sartori et al. (2001) observaram que não houve efeito da temperatura ambiente sobre a composição dos tipos de fibras no músculo flexor longo do hálux de frangos de corte. Este fato pode estar relacionado à variação no consumo de ração, em função das alterações na temperatura ambiente, quente ou fria. Para testar a hipótese de que a temperatura é um fator que por si só pode alterar a composição dos tipos de fibras musculares, é necessário que o efeito do consumo de ração seja controlado.

O objetivo deste trabalho foi avaliar o efeito da temperatura ambiente sobre as características morfométricas dos tipos de fibras musculares do músculo flexor longo do hálux de frangos de corte machos submetidos à mesma quantidade de ração (pair-feeding), isolando-se o efeito do consumo voluntário de ração.

\section{Material e Métodos}

Foram utilizados 16 frangos de corte machos da linhagem Ross, obtidos de ovos de matrizes com 47 semanas de idade, submetidas às mesmas condições de ambiente, manejo e alimentação. Os ovos foram incubados em uma mesma incubadora e o nascimento ocorreu em um único nascedouro. Após o nascimento, os pintos foram sexados pela asa, selecionados e vacinados contra as doenças de Marek, Gumboro e Bouba Aviária.

As aves foram criadas individualmente em gaiolas do 5 o ao 43 o dia de idade (período experimental). Sete aves foram mantidas em uma câmara aquecida de 3 a $9^{\circ} \mathrm{C}$ acima da termoneutralidade, de acordo com a idade da ave, isto é, 35,34 e $33^{\circ} \mathrm{C}$ do $5 \underline{0}$ ao $7 \underline{0}$, $8 \underline{\mathrm{o}}$ ao $16^{\mathrm{o}}$ e $17 \underline{\mathrm{O}}$ ao $42 \underline{\mathrm{o}}$ dia de idade, respectivamente. Nove aves foram mantidas em outra câmara resfriada, entre 7 e $9^{\circ} \mathrm{C}$ abaixo da termoneutralidade, isto é, 25 , $21,19,17$ e $15^{\circ} \mathrm{C}$ do $5^{\circ}$ ao $7 \stackrel{\text { o }}{8} 8^{\circ}$ a a 14 o, 15 o ao $16^{\circ}$, $17 \underline{0}$ ao $21 \underline{\mathrm{o}}$ e $22 \underline{\mathrm{o}}$ ao $42 \underline{\mathrm{o}}$ dia de idade, respectivamente.

Foram fornecidas 24 horas de luz em ambas as câmaras. A água foi fornecida à vontade em bebedouros tipo "niple". As aves foram alimentadas com ração farelada em cochos individuais. O programa nutricional foi dividido em três fases: inicial (1-21

R. Bras. Zootec., v.32, n.4, p.918-925, 2003 
dias), crescimento (22-35 dias) e final (36-42 dias), com rações contendo 3050,3150 e $3200 \mathrm{kcal} / \mathrm{kg}$ de energia metabolizável aparente e 21, 19 e $18 \%$ de proteína bruta, respectivamente. As aves da câmara aquecida receberam ração à vontade durante todo o período experimental. As aves da câmara fria receberam quantidade restrita de ração, correspondente ao consumo das aves da câmara aquecida (pairfeeding). A quantidade de ração fornecida diariamente para as aves da câmara fria foi ajustada diariamente, acrescentando-se certa quantidade, em função da medida do consumo do dia anterior das aves da câmara aquecida.

Aos 43 dias de idade, os frangos foram sacrificados por deslocamento cervical e o músculo flexor longo do hálux da perna direita foi dissecado imediatamente e pesado sem tendões e sem gordura. Fez-se um corte transversal na região mediana do músculo, dividindo-o em duas partes. A parte distal (onde fica o tendão, próxima à articulação tibiotarsotarsometatarsiana) foi colhida e imediatamente congelada durante dois minutos em N-Hexana, previamente resfriada a $-70^{\circ} \mathrm{C}$ em $\mathrm{N}_{2}$ líquido (Chayen et al., 1969). Os fragmentos foram identificados e acondicionados em botijão de $\mathrm{N}_{2}$ líquido para conservação das amostras.

Em um micrótomo-criostato a $-20^{\circ} \mathrm{C}$ foram obtidas quatro séries de cortes histológicos com $8 \mathrm{~mm}$ de espessura. As séries de cortes foram submetidas às seguintes técnicas: 1) Hematoxilina-Eosina (HE), que permite analisar a morfologia das fibras musculares, ou seja, sua arquitetura, forma, tamanho, posição dos núcleos, presença de artefatos ou anomalias, mas não permite diferenciação dos tipos de fibras; 2) demonstração da atividade da Nicotinamida Adenina Tetrazólio Redutase (NADH-TR), que indica a presença da atividade oxidativa através de grânulos corados em azul (finos agregados de formazana) que se depositam nos locais onde estão as mitocôndrias, conforme a técnica de Pearse (1972), modificada por Dubowitz \& Brooke (1984); 3) demonstração da atividade ATPase miofibrilar (m-ATPase), utilizando-se o método de Dubowitz \& Brooke (1984), após pré-incubação em meio alcalino $(\mathrm{pH} \mathrm{10,4)} \mathrm{e} \mathrm{em} \mathrm{meio} \mathrm{ácido}$ ( $\mathrm{pH} 4,6$ ), que fornece indicação da velocidade de contração da fibras. Para a nomenclatura dos tipos de fibra, foram adotados os critérios de Peter et al. (1972).

O tamanho da fibra foi avaliado pela medida do seu menor diâmetro (Dubowitz \& Broke, 1984). As medidas de número e tamanho dos tipos de fibras foram obtidas de cortes que reagiram com a m-ATPase após pré-incubação ácida $(\mathrm{pH} 4,6)$, pois esta reação permitiu a diferenciação dos três tipos de fibras. Dos cortes presentes em cada lâmina, foi escolhido o mais íntegro e o de melhor padrão de reação. As reações de NADH-TR e m-ATPase após pré-incubação alcalina serviram como controle para a identificação dos tipos de fibras.

Para contagem, estabelecimento das freqüências (\%) e obtenção do menor diâmetro dos tipos de fibras, dez áreas de $36.970 \mu \mathrm{m}^{2}$ foram analisados ao acaso por corte escolhido/ave, utilizando-se um microscópio ótico comum acoplado a um analisador de imagens (Videoplan - OPTIMAS - USA) e a um computador. Foram contadas e medidas as fibras que se encontravam dentro das áreas.

Para obtenção da área do corte transversal do músculo, foi utilizada uma lupa acoplada ao analisador de imagens (Videoplan - OPTIMAS - USA) e ao computador. O número dos diferentes tipos de fibras no músculo foi obtido multiplicando-se o número médio dos tipos de fibras por área analisada pela área da seção transversal do músculo e dividido-se pela área analisada (Aberle \& Stewart, 1983). O número total de fibras no músculo foi obtido pela soma do número dos tipos de fibras.

A análise estatística das características avaliadas (peso vivo ao abate, peso do músculo, relação peso do músculo/peso vivo, área da seção transversal do músculo e freqüência, tamanho e número dos tipos de fibras) foi feita pelo método da análise de variância para um delineamento inteiramente casualizado com dois tratamentos (temperatura acima e abaixo da termoneutralidade) e com número desigual de repetições, 7 e 9 respectivamente, utilizando-se o procedimento General Linear Model (GLM) do Statistical Analysis System (SAS, 1996).

\section{Resultados e Discussão}

Nas Figuras 1 e 2, estão mostradas as fibras musculares nos cortes histológicos do músculo flexor longo do hálux, submetidos aos quatro tipos de reação, para as aves criadas no ambiente quente e frio em "pair-feeding", respectivamente.

Nos cortes do músculo flexor longo do hálux submetidos à reação com a m-ATPase após préincubação alcalina (pH 10,4), as fibras do tipo FG

R. Bras. Zootec., v.32, n.4, p.918-925, 2003 
reagiram intensamente e as fibras dos tipos FOG e SO apresentaram intensidade de reação fraca a moderada, de forma que não foi possível diferenciá-las com esta coloração. Na reação da m-ATPase após pré-incubação ácida ( $\mathrm{pH} 4,6)$, as fibras do tipo $\mathrm{SO}$ reagiram intensamente, as fibras do tipo $\mathrm{FG}$ reagiram fracamente e as fibras do tipo FOG reagiram moderadamente, apresentando intensidade de coloração intermediária entre $\mathrm{SO}$ e $\mathrm{FG}$, permitindo a diferenci-
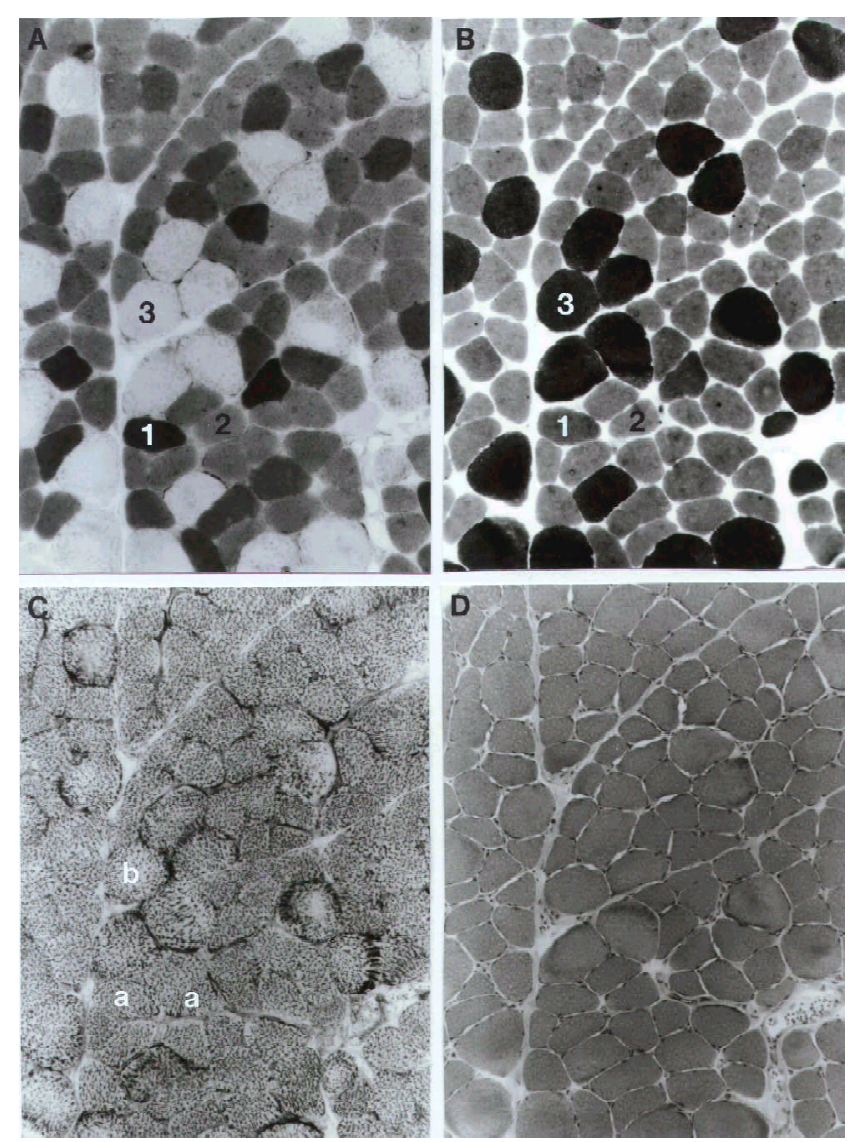

Figura 1 - Cortes transversais do músculo flexor longo do hálux de frangos de corte machos aos 43 dias de idade submetidos ao estresse pelo calor ( $\mathrm{A}=\mathrm{m}$-ATPase, após pré-incubação em $\mathrm{pH} 4,6$; $\mathrm{B}=\mathrm{m}$-ATPase, após pré-incubação em $\mathrm{pH}$ $10,4 ; \mathrm{C}=\mathrm{NADH}-\mathrm{TR}$ e $\mathrm{D}=\mathrm{HE}) .1$ = SO, 2 = FOG e $3=$ FG. a = metabolismo oxidativo intenso e $\mathrm{b}=$ metabolismo oxidativo moderado. 400x.

Figure 1 - Cross section of flexor hallucis longus muscle of 43-day-old male broilers subjected to heat stress $(A=m$-ATPase, $p H$ 4,6; $B=m$-ATPase, $p H$ 10,4; $C$ $=N A D H-T R$ and $D=H E) .1=S O, 2=F O G$ e $3=F G$. $a=$ intense oxidative metabolism and $b=$ moderate oxidative metabolism. $x 400$. ação dos três tipos de fibras. Na reação com a NADH-TR, observa-se que as fibras de metabolismo oxidativo (SO e FOG) reagiram intensamente e fibras de metabolismo glicolítico (FG) reagiram moderadamente, indicando que todas as fibras deste músculo apresentavam predomínio de caráter oxidativo (Figuras 1 e 2).

$\mathrm{Na}$ Tabela 1, são mostrados os dados médios de peso vivo e morfometria do músculo flexor longo do
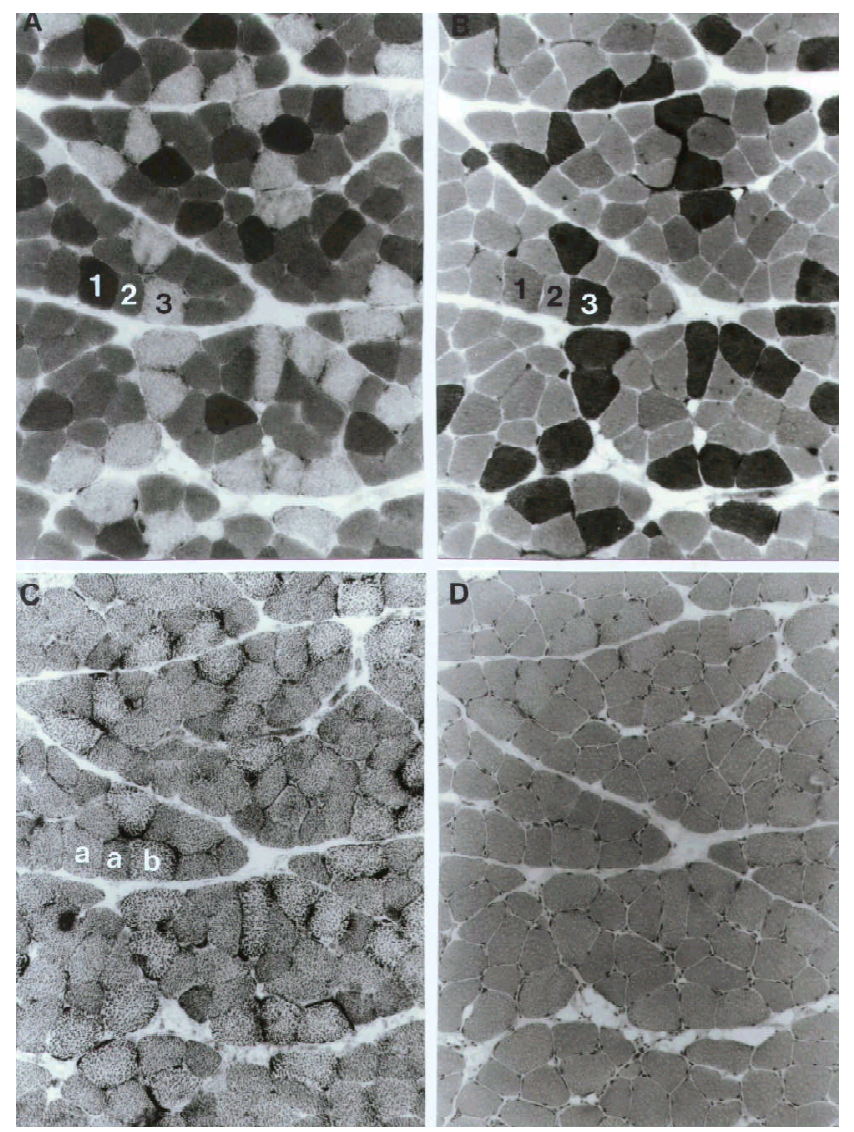

Figura 2 - Cortes transversais do músculo flexor longo do hálux de frangos de corte machos aos 43 dias de idade submetidos ao estresse pelo frio $(A=m-A T P a s e$, após pré-incubação em $\mathrm{pH} 4,6 ; \mathrm{B}=\mathrm{m}$-ATPase, após pré-incubação em $\mathrm{pH} 10,4 ; \mathrm{C}=\mathrm{NADH}-\mathrm{TR}$ e $\mathrm{D}=\mathrm{HE}) .1$ = SO, $2=\mathrm{FOG} \mathrm{e} 3=\mathrm{FG} \cdot \mathrm{a}=$ metabolismo oxidativo intenso $\mathrm{e} \mathrm{b}=$ metabolismo oxidativo moderado. 400x.

Figure 2 - Cross section of flexor hallucis longus muscle of 43-day-old male broilers subjected to cold stress $(A=m$-ATPase, $p H 4,6 ; B=m$-ATPase, $p H$ 10,4; C $=N A D H-T R$ and $D=H E) .1=S O, 2=F O G$ e $3=F G$. $a=$ intense oxidative metabolism and $b=$ moderate oxidative metabolism. $x 400$. 
Tabela 1 - Peso vivo (PV), peso (PM) e área da seção transversal (AM) do músculo flexor longo do hálux e relação entre peso do músculo e peso vivo (PM/PV) de frangos de corte machos com 43 dias de idade, alimentados em "pairfeeding", segundo a temperatura ambiente

Table 1 - Body weight (BW), flexor hallucis longus muscle weigth (MW) and cross section area (MA) and muscle weigth:body weigth ratio (MW/BW) of 43-day-old male broilers in pair-feeding, according to the environmental temperature

\begin{tabular}{lcccc}
\hline $\begin{array}{l}\text { Temperatura } \\
\text { Temperature }\end{array}$ & $\begin{array}{c}\mathrm{PV}, \mathrm{g} \\
\mathrm{BW}, \mathrm{g}\end{array}$ & $\begin{array}{c}\mathrm{PM}, \mathrm{g} \\
\mathrm{MW}, \mathrm{g}\end{array}$ & $\begin{array}{c}\mathrm{AM}, \mathrm{mm}^{2} \\
M A, \mathrm{~mm}^{2}\end{array}$ & $\begin{array}{c}\mathrm{PM} / \mathrm{PV} \\
M W / B W\end{array}$ \\
\hline $\begin{array}{l}\text { Quente }^{1} \\
\text { Hot }\end{array}$ & 1255 & 0,938 & 40,20 & 0,075 \\
Frio $^{2}$ & 1087 & 0,844 & 38,42 & 0,078 \\
Cold & & & & \\
$\mathrm{F}$ & 11,16 & 3,98 & 0,35 & 0,42 \\
$\mathrm{P}$ & 0,005 & 0,066 & 0,566 & 0,529 \\
$\mathrm{DP}^{3}$ & 100 & 0,094 & 6,02 & 0,008 \\
SD & & & & \\
$\mathrm{CV}(\%)^{3}$ & 8,65 & 10,60 & 15,35 & 10,12 \\
\hline
\end{tabular}

${ }^{1} \mathrm{~N}=9$

$2 \mathrm{~N}=7$.

hálux da perna direita dos frangos de corte. Constam nas Tabelas 2 e 3 os valores médios de diâmetro e freqüência dos tipos de fibras musculares nas regiões superficial e profunda do músculo, respectivamente. Nas Tabelas 4 e 5, estão apresentados os valores médios de diâmetro, freqüência e número dos tipos de fibras no músculo, respectivamente.

Aos 43 dias de idade, o peso vivo das aves criadas na câmara aquecida foi maior $(\mathrm{P}<0,05)$ que o das aves criadas na câmara fria, as quais apresentaram pesos corporais médios $13,5 \%$ menor. Frangos criados no ambiente quente apresentaram, em média, $1255 \mathrm{~g}$ de peso corporal aos 43 dias de idade, $49,9 \%$ menor que o peso indicado pelo manual da linhagem (Agross, 1997) para a mesma idade (2504 g). Contudo, comparando-se frio e calor, não houve efeito $(\mathrm{P}>0,05)$ da temperatura para peso e área da seção transversal do músculo e relação peso do músculo/peso vivo (Tabela 1).

O menor peso observado nos frangos criados no ambiente quente em relação ao peso indicado pelo manual da linhagem (Agross, 1997) para a mesma idade está de acordo com os observados por outros pesquisadores (Teeter et al., 1985; May et al., 1998), que observaram queda acentuada no ganho de peso dos frangos de corte em condições de estresse agudo pelo calor. Embora não tenha havido efeito significativo da temperatura sobre o tamanho do músculo, pode-se observar tendência de maiores valores para peso e área da seção transversal do músculo nas aves submetidas ao calor.

Em ambientes frios e em condições normais de alimentação, com fornecimento de alimento à vontade, os frangos compensam a maior exigência de energia para manutenção da temperatura cor-

Tabela 2 - Diâmetro $(\mu \mathrm{m})$ dos tipos de fibras SO, FOG e FG nas regiões superficial e profunda do músculo flexor longo do hálux de frangos de corte machos com 43 dias de idade, alimentados em pair-feeding, segundo a temperatura ambiente ${ }^{1}$

Table 2 - Diameter $(\mu \mathrm{m})$ of SO, FOG and FG fiber types in the superficial and deep regions in the flexor hallucis longus muscle of 43-day-old male broilers in pair-feeding, according to the environmental temperature

\begin{tabular}{|c|c|c|c|c|c|c|}
\hline \multirow[t]{2}{*}{$\begin{array}{l}\text { Temperatura } \\
\text { Temperature }\end{array}$} & \multicolumn{3}{|c|}{$\begin{array}{l}\text { Região superficial } \\
\text { Superficial region }\end{array}$} & \multicolumn{3}{|c|}{$\begin{array}{l}\text { Região profunda } \\
\text { Deep region }\end{array}$} \\
\hline & SO & FOG & FG & SO & FOG & FG \\
\hline $\begin{array}{l}\text { Quente } \\
\text { Hot }\end{array}$ & 30,19 & 29,23 & 49,66 & 37,45 & 35,80 & 47,81 \\
\hline $\begin{array}{l}\text { Frio } \\
\text { Cold }\end{array}$ & 37,27 & 31,29 & 39,36 & 35,68 & 29,91 & 35,41 \\
\hline $\mathrm{F}$ & 4,20 & 0,27 & 15,84 & 0,36 & 2,48 & 16,73 \\
\hline $\begin{array}{l}\mathrm{P} \\
\mathrm{DP}^{2}\end{array}$ & $\begin{array}{l}0,060 \\
6,85\end{array}$ & $\begin{array}{l}0,614 \\
7,92\end{array}$ & $\begin{array}{r}0,001 \\
5,14\end{array}$ & $\begin{array}{l}0,559 \\
5,88\end{array}$ & $\begin{array}{l}0,138 \\
7,42\end{array}$ & $\begin{array}{r}0,001 \\
6,02\end{array}$ \\
\hline$S D$ & & & & & & \\
\hline $\mathrm{CV}(\%)^{2}$ & 20,05 & 26,08 & 11,71 & 16,14 & 22,84 & 14,74 \\
\hline
\end{tabular}

1 Valores correspondentes à média de cinco áreas de $36.970 \mu \mathrm{m}^{2}$, analisados ao acaso por região da seç̧ão transversal do músculo por ave.

1 The values are the average of five $36,970 \mu \mathrm{m}^{2}$ areas that were analyzed at random per tranverse section of a region of broiler muscle.

$2 \mathrm{DP}=$ desvio-padrão; $\mathrm{CV}=$ coeficiente de variação.

$2 S D=$ standard deviation; $C V=$ coefficient of variation .

R. Bras. Zootec., v.32, n.4, p.918-925, 2003 
poral aumentando o consumo de alimento (NRC, 1994). Como ambos os grupos receberam a mesma quantidade de ração, os menores pesos das aves no frio indicam que maior parte da energia da dieta foi desviada para produção de calor e manutenção da temperatura corporal, em detrimento do crescimento. O efeito compensatório do maior consumo de ração em aves submetidas a condições de temperatura ambiental fria foi eliminado, já que as aves tiveram acesso restrito à ração. Al-Harthi \& Macleod (1996), comparando aves mantidas em ambiente aquecido $\left(30^{\circ} \mathrm{C}\right)$ e na termoneutralidade $\left(20^{\circ} \mathrm{C}\right)$ alimentados em pairfeeding, não observaram diferenças de ganho de peso entre as aves, confirmando que o consumo de alimento é um fator muito importante que afeta essa característica.

Analisando-se as regiões superficial e profunda do músculo flexor longo do hálux, verificou-se que não houve efeito da temperatura ambiente para o diâmetro das fibras do tipo SO e FOG. Entretanto, as fibras do tipo FG apresentaram maior diâmetro $(\mathrm{P}<0,05)$ nas aves criadas em ambiente quente que no frio, tanto na região superficial como na região profunda do músculo (Tabela 2). A temperatura não afetou $(\mathrm{P}>0,05)$ a freqüência dos tipos de fibras nas regiões superficial e profunda do músculo (Tabela 3 ).

Quando se considerou o músculo como um todo, independentemente das suas regiões, o diâmetro das fibras do tipo $\mathrm{FG}$ foi maior $(\mathrm{P}<0,05)$ no músculo das aves mantidas na câmara aquecida. Para as fibras do tipo SO e FOG, não houve efeito da temperatura sobre o diâmetro. A média do diâmetro dos três tipos de fibras também não foi afetada pela temperatura ambiente. Também não se observou efeito significativo da temperatura ambiente sobre as freqüências dos tipos de fibras (Tabela 4) e sobre o número dos tipos de fibras no músculo (Tabela 5). O número total de fibras no músculo flexor longo do hálux não foi afetado $(\mathrm{P}>0,05)$ pela temperatura ambiente (Tabela 5).

Embora vários autores (Ingram \& Dauncey, 1986; Dauncey \& Ingram, 1988; Duchamp et al., 1992) tenham observado que a baixa temperatura e/ou o baixo nível de ingestão de energia possam alterar a composição de fibras musculares, aumentando a freqüência e o diâmetro das fibras de metabolismo oxidativo ( $\mathrm{SO}$ e FOG), isto não foi observado neste experimento. Contudo, as aves submetidas ao frio apresentaram diminuição no diâmetro das fibras de metabolismo glicolítico (FG), enquanto as fibras de metabolismo oxidativo ( $\mathrm{SO}$ e FOG) não tiveram alteração no seu diâmetro quando comparadas às submetidas ao calor com alimentação ad libitum. Esta preservação seletiva no tamanho das fibras oxidativas em animais submetidos ao frio também foi observada por Harrison et al. (1996), em suínos, que atuariam como um mecanismo de conservação de energia nos

Tabela 3 - Freqüência (\%) dos tipos de fibras SO, FOG e FG nas regiões superficial e profunda do músculo flexor longo do hálux de frangos de corte machos com 43 dias de idade, alimentados em pair-feeding, segundo a temperatura ambiente ${ }^{1}$

Table 3 - Frequency (\%) of SO, FOG and FG fiber types in the superficial and deep regions in the flexor hallucis longus muscle of 43-day-old male broilers in pair-feeding, according to the environmental temperature

\begin{tabular}{|c|c|c|c|c|c|c|}
\hline \multirow[t]{2}{*}{$\begin{array}{l}\text { Temperatura } \\
\text { Temperature }\end{array}$} & \multicolumn{3}{|c|}{$\begin{array}{l}\text { Região superficial } \\
\text { Superficial region }\end{array}$} & \multicolumn{3}{|c|}{$\begin{array}{l}\text { Região profunda } \\
\text { Deep region }\end{array}$} \\
\hline & SO & FOG & FG & $\mathrm{SO}$ & FOG & FG \\
\hline $\begin{array}{l}\text { Quente } \\
\text { Hot }\end{array}$ & 23,44 & 49,37 & 27,20 & 25,55 & 55,48 & 18,97 \\
\hline $\begin{array}{l}\text { Frio } \\
\text { Cold }\end{array}$ & 21,81 & 45,38 & 32,81 & 27,20 & 49,20 & 23,59 \\
\hline $\mathrm{F}$ & 0,67 & 1,24 & 4,19 & 0,18 & 2,72 & 1,21 \\
\hline$P$ & 0,425 & 0,285 & 0,060 & 0,675 & 0,121 & 0,291 \\
\hline $\begin{array}{l}\mathrm{DP}^{2} \\
S D\end{array}$ & 3,94 & 7,11 & 5,44 & 7,66 & 7,55 & 8,36 \\
\hline $\mathrm{CV}(\%)^{2}$ & 17,49 & 15,10 & 17,94 & 28,93 & 14,53 & 38,77 \\
\hline
\end{tabular}

${ }_{1}^{1}$ Valores correspondentes à média de cinco áreas de $36.970 \mu \mathrm{m}^{2}$, analisados ao acaso por região da secção transversal do músculo por ave.

1 The values are the average of five $36,970 \mu \mathrm{m}^{2}$ areas that were analyzed at random per tranverse section of a region of broiler muscle.

$2 \mathrm{DP}=$ desvio-padrão; CV = coeficiente de variação.

${ }^{2} S D=$ standard deviation; $C V=$ coefficient of variation .

R. Bras. Zootec., v.32, n.4, p.918-925, 2003 
Tabela 4 - Diâmetro $(\mu \mathrm{m})$ e freqüência (\%) dos tipos de fibras SO, FOG e FG no músculo flexor longo do hálux de frangos de corte machos com 43 dias de idade, alimentados em pair-feeding, segundo a temperatura ambiente ${ }^{1}$

Table 4 - Diameter $(\mu \mathrm{m})$ and frequency (\%) of SO, FOG and FG fiber types in the flexor hallucis longus muscle of 43-day-old male broilers in pair-feeding, according to the environmental temperature

\begin{tabular}{|c|c|c|c|c|c|c|c|}
\hline \multirow[t]{2}{*}{$\begin{array}{l}\text { Temperatura } \\
\text { Temperature }\end{array}$} & \multicolumn{3}{|c|}{$\begin{array}{c}\text { Diâmetro }(\mu \mathrm{m}) \\
\text { Diameter }\end{array}$} & \multirow[b]{2}{*}{ Total } & \multicolumn{3}{|c|}{$\begin{array}{c}\text { Freqüência (\%) } \\
\text { Frequency }\end{array}$} \\
\hline & $\mathrm{SO}$ & $\overline{\text { FOG }}$ & FG & & $\mathrm{SO}$ & FOG & FG \\
\hline $\begin{array}{l}\text { Quente } \\
\text { Hot }\end{array}$ & 33,22 & 31,29 & 48,69 & 37,74 & 24,42 & 52,76 & 22,82 \\
\hline $\begin{array}{l}\text { Frio } \\
\text { Cold }\end{array}$ & 36,28 & 30,36 & 37,28 & 34,64 & 24,40 & 47,67 & 27,93 \\
\hline $\begin{array}{l}\mathrm{F} \\
\mathrm{P}\end{array}$ & $\begin{array}{c}0,99 \\
0,336\end{array}$ & $\begin{array}{c}0,07 \\
0,796\end{array}$ & $\begin{array}{l}23,32 \\
0,003\end{array}$ & $\begin{array}{c}1,53 \\
0,237\end{array}$ & $\begin{array}{c}0,00 \\
0,994\end{array}$ & $\begin{array}{c}3,63 \\
0,078\end{array}$ & $\begin{array}{l}2,58 \\
0,130\end{array}$ \\
\hline $\begin{array}{l}\text { D.P. }{ }^{2} \\
\text { S.D. } \\
\text { CV }(\%)^{2}\end{array}$ & 6,10 & 7,00 & 4,69 & 4,96 & 3,93 & 5,30 & 6,31 \\
\hline
\end{tabular}

1 Valores correspondentes à média de dez áreas de $36.970 \mu \mathrm{m}^{2}$, analisados ao acaso por região da secção transversal do músculo por ave.

1 The values are the average of ten $36,970 \mu \mathrm{m}^{2}$ areas that were analyzed at random per tranverse section of a region of broiler muscle.

$2 \mathrm{DP}=$ desvio-padrão; $\mathrm{CV}=$ coeficiente de variação.

$2 S D=$ standard deviation; $C V=$ coefficient of variation.

Tabela 5 - Número dos tipos de fibras SO, FOG e FG no músculo flexor longo do hálux de frangos de corte machos com 43 dias de idade, alimentados em pair-feeding, segundo a temperatura ambiente ${ }^{1}$

Table 5 - Number of SO, FOG and FG fiber types in the flexor hallucis longus muscle of 43-day-old male broilers in pair-feeding, according to the environmental temperature

\begin{tabular}{lcccc}
\hline $\begin{array}{l}\text { Temperatura } \\
\text { Temperature }\end{array}$ & SO & FOG & FG & $\begin{array}{l}\text { Total } \\
\text { Total }\end{array}$ \\
\hline $\begin{array}{l}\text { Quente } \\
\text { Hot }\end{array}$ & 4330 & 9279 & 4095 & 17703 \\
$\begin{array}{l}\text { Frio } \\
\text { Cold }\end{array}$ & 4580 & 9238 & 5398 & 19216 \\
F & 0,32 & 0,00 & 2,76 & 0,67 \\
P & 0,580 & 0,971 & 0,119 & 0,427 \\
DP $^{2}$ & 874 & 2156 & 1556 & 3670 \\
SD & 19,56 & 23,29 & 32,22 & 19,78 \\
CV $(\%)^{2}$ & & & &
\end{tabular}

10 número dos tipos de fibras foi obtido multiplicando-se o número médio dos tipos de fibras por área analisada pela área da secção transversal do músculo e dividido-se pela área analisada $\left(36.970 \mu \mathrm{m}^{2}\right)$ (The number of fiber types refers to the average number of fibre type peranalyzed area times the transverse sectional area of the muscle divided by the area $\left(36,970 \mu \mathrm{m}^{2}\right)$.

$2 \mathrm{DP}=$ desvio-padrão; $\mathrm{CV}=$ coeficiente de variação

$2 S D=$ standard deviation; $C V=$ coefficient of variation.

animais submetidos ao frio.

O estudo demonstra que situações mais agressivas, como baixa ingestão de alimento associadas a temperatura ambiente fria $\left(7\right.$ a $9^{\circ} \mathrm{C}$ abaixo da termoneutralidade), podem promover queda muito acentuada no crescimento dos frangos de corte e alteração na composição de fibras no músculo flexor longo do hálux. As fibras glicolíticas (FG) foram as mais afetadas e diminuíram de tamanho, enquanto os tamanhos das fibras com metabolismo oxidativo (FOG e SO) foram preservados. Esses resultados confirmaram que o crescimento muscular é dependente, principalmente, da hipertrofia das fibras glicolíticas que, por sua vez, depende do aporte nutritivo para o crescimento. Por outro lado, a necessidade de produção de calor, para a manutenção da temperatura corporal, parece depender da preservação estrutural das fibras oxidativas.

\section{Conclusões}

Nas condições deste experimento, pode-se concluir que o estresse pelo frio $\left(7\right.$ a $9{ }^{\circ} \mathrm{C}$ abaixo da termoneutralidade), associado à limitação no consumo de alimento (pair-feeding), reduziu o crescimento dos frangos de corte, promovendo alteração na composição dos tipos de fibras no músculo flexor longo do hálux, com redução do tamanho das fibras do tipo FG e manutenção do tamanho das fibras $\mathrm{SO}$ e FOG, as quais apresentam metabolismo oxidativo, associado à maior produção de calor.

\section{Literatura Citada}

ABERLE, E.D.; STEWART, T.S. Growth of fiber types and apparent fiber number in skeletal muscle of broiler- and layertype chickens. Growth, v.47, p.135-144, 1983.

AGROCERES ROSS MELHORAMENTO GENÉTICO DE AVES S.A. - AGROSS. Manual de manejo de frangos de

R. Bras. Zootec., v.32, n.4, p.918-925, 2003 
corte. Rio Claro: 1997. $8 \mathrm{p}$

AL-HARTHI, M.A.; MACLEOD, M.G. Analysis of heat stress effects on growth by pair-feeding. British Poultry Science, v.37, p.S42-S43, 1996.

BANKS, W.J. Tecido muscular. In: BANKS, W.J. (Ed.) Histologia veterinária aplicada. 2.ed. São Paulo: Manole Ltda, 1992. p.215-236.

BALLANTYNE, J.S.; GEORGE, J.C. An ultrastructural and histological analysis of the effects of cold acclimation on vertebrate skeletal muscle. Journal of Thermal Biology, v.3, p.109-116, 1978.

CHAMBERS, J.R.; FORTIN, A.; MACKIE, D.A. et al. Comparison of sensory properties of meat from broilers of modern stocks and experimental strains differing in growth and fatness. Canadian Institute of Food Science and Technology Journal, v.22, p.353-358, 1989.

CHAYEN, J.; BITENSKY, L.; BUTCHER, R.G. et al. A guide to pratical histochemstry. London: Oliver \& Bould, 1969. $261 \mathrm{p}$.

DAUNCEY, M.J.; INGRAM, D.L. Influence of environmental temperature and energy intake on skeletal muscle respiratory enzimes and morphology. European Journal of Applied Physiology, v.58, p.239-244, 1988.

DAUNCEY, M.J.; GILMOUR, R.S. Regulatory factors in the control of muscle development. Proceedings of the Nutrition Society, v.55, p.543-559, 1996.

DUBOWITZ, V.; BROOKE, M. Muscle biopsy: a modern approach. London: W.B. Saunders Company, 1984. 472p.

DUCHAMP, C.; COHEN-ADAD, F.; ROUANET, J.L. et al. Histochemical arguments for muscular non-shivering thermogenesis in muscovy ducklings. Journal of Physiology, v.457, p.27-45, 1992.

HARRISON, A.P.; ROWLERSON, A.M.; DAUNCEY, M.J. Selective regulation of myofiber differentiation by energy status during postnatal development. American Journal of Physiology, v.270, p.667-674, 1996.

INGRAM, D.L.; DAUNCEY, M.J. Environmental effects on growth and development. In: BUTTERY, P.J.; LINDSAY,
D.B.; HAYNES, N.B. (Ed.). Control and manipulation of animal growth. London: Butterworths, 1986. p.5-20.

MAY, J.D.; LOTT, B.D.; SIMMONS, J.D. The effect of environmental temperature and body weight on growth rate and feed:gain of male broilers. Poultry Science, v.77, p.499$501,1998$.

NATIONAL RESEARCH COUNCIL - NRC. Nutrient requeriment of poultry. 9.ed. Washington, D.C.: National Academic Press, 1994. 155p.

NEWCOMBE, M. Tendências mundiais no manejo de frangos de corte. In: CONFERÊNCIA APINCO DE CIÊNCIA E TECNOLOGIA AVÍCOlAS, 1994, Santos. Anais... Santos: FACTA, 1994. p.1-2.

PETER, J.B.; BARNARD, R.J.; EDGERTON, V.R. et al. Metabolic profiles of three fiber types of skeletal muscle in Guinea pig and rabbits. Biochemistry, v.11, p.26272633, 1972.

ROENIGK, W.P. Keynote address: world poultry consumption. Poultry Science, v.78, p.722-728, 1999.

SAMS, A.R. Meat quality during processing. Poultry Science, v.78, p.798-803, 1999.

SARTORI, J.R.; GONZALES, E.; DAL PAI, V. et al. Efeito da temperatura ambiente e da restrição alimentar sobre o desempenho e composição de fibras musculares esqueléticas de frangos de corte. Revista Brasileira de Zootecnia, v.30, p.1779-1790, 2001.

SAS INSTITUTE. SAS/STAT user's guide. 4.ed. Version 6.11. Cary: 1996. 842p.

TEETER, R.G.; SMITH, F.N.; OWENS, F.N. et al. Chronic heat stress and respiratory alkalosis: occurrence and treatment in broiler chicks. Poultry Science, v.64, p.1060-1064, 1985.

Recebido em: 09/04/02 Aceito em: 09/12/02 